\title{
Ulam stability for nonautonomous quantum equations
}

\author{
Douglas R. Anderson ${ }^{1}$ and Masakazu Onitsuka ${ }^{2^{*}}$
}

${ }^{*}$ Correspondence:

onitsuka@xmath.ous.ac.jp

2Department of Applied

Mathematics, Okayama University

of Science, Okayama, 700-0005,

Japan

Full list of author information is

available at the end of the article

\begin{abstract}
We establish the Ulam stability of a first-order linear nonautonomous quantum equation with Cayley parameter in terms of the behavior of the nonautonomous coefficient function. We also provide details for some cases of Ulam instability.
\end{abstract}

MSC: 39A06; 39A13; 39A30; 34N05

Keywords: Ulam stability; Quantum equations; q-Difference equations; Dynamic equations; Nonautonomous equations; Variable coefficient

\section{Introduction}

In a series of recent papers [7-9] the authors introduced the study of Ulam stability for linear quantum ( $q$-difference) equations of first order with a complex constant coefficient. See $[1,2,19]$ for the literature on related topics. As yet, there are no works in the literature dealing with first-order linear quantum equations with nonautonomous (variable) coefficient functions, which we initiate below.

As one of the stability types of functional equations, Ulam (or Hyers-Ulam) stability has been investigated by many researchers. Since the paper is devoted to a highly active domain with a plethora of interesting and applicable results, we must pay attention to more classical and recent results in the fields of functional equations. For example, for some highly important works, we direct the reader to [3, 4, 20, 22, 24, 27-29, 31-33, 42, 46]. We also draw attention to the books $[5,21,23,25,30,43,44]$.

Since Popa $[39,40]$ began studying the Ulam stability of linear difference equations (linear recurrences) in 2005, many researchers have investigated this problem; for example, see $[6,10,15,16,37,38,41,45]$. For higher-order difference equations, see [13, 14], and for nonlinear difference equations, see [26, 34-36]. As of yet, the results for variable coefficients are very few, even for difference equations. For the latest studies on the Ulam stability related to variable and periodic coefficients, see [11, 17, 18]. For results on the Ulam stability for a first-order linear difference equation with nonconstant coefficients in Banach spaces, with the best Ulam constant, see [12].

Let $\mathbb{N}$ be the set of natural numbers, and let $\mathbb{N}_{0}:=\mathbb{N} \cup\{0\}$. Define the quantum set

$$
q^{\mathbb{N}_{0}}:=\left\{1, q, q^{2}, q^{3}, \ldots\right\}
$$

(c) The Author(s) 2021. This article is licensed under a Creative Commons Attribution 4.0 International License, which permits use, sharing, adaptation, distribution and reproduction in any medium or format, as long as you give appropriate credit to the original author(s) and the source, provide a link to the Creative Commons licence, and indicate if changes were made. The images or other third party material in this article are included in the article's Creative Commons licence, unless indicated otherwise in a credit line to the material. If material is not included in the article's Creative Commons licence and your intended use is not permitted by statutory regulation or exceeds the permitted use, you will need to obtain permission directly from the copyright holder. To view a copy of this licence, visit http://creativecommons.org/licenses/by/4.0/. 
for $q>1$. In this paper, we consider the nonautonomous Cayley quantum equation

$$
D_{q} z(t)=\alpha(t)\langle z(t)\rangle_{\beta}, \quad t \in q^{\mathbb{N}_{0}},
$$

where $\alpha(t)$ is a complex-valued time-varying coefficient, the $q$-difference operator is

$$
D_{q} z(t):=\frac{z(q t)-z(t)}{(q-1) t}, \quad q>1,
$$

and the Cayley component is

$$
\left\langle\left. z(t)\right|_{\beta}:=\beta z(q t)+(1-\beta) z(t), \quad 0 \leq \beta \leq 1 .\right.
$$

If $\beta=0$, then the Cayley quantum equation reduces to the mere quantum equation

$$
D_{q} z(t)=\alpha(t) z(t), \quad t \in q^{\mathbb{N}_{0}} .
$$

It is well known that $D_{q} z(t) \rightarrow z^{\prime}(t)$ as $q \searrow 1$, so we can say that the quantum equation is an approximate equation of the differential equation $z^{\prime}(t)=\alpha(t) z(t)$. Notice that equation (1.1) can be rewritten as

$$
[1-\beta(q-1) t \alpha(t)] z(q t)=[1+(1-\beta)(q-1) t \alpha(t)] z(t) .
$$

This formula shows that the condition

$$
1-\beta(q-1) t \alpha(t) \neq 0 \neq 1+(1-\beta)(q-1) t \alpha(t) \quad \text { for } t \in q^{\mathbb{N}_{0}}
$$

is necessary to keep the recurrence viable. For this reason, we assume this condition throughout this paper.

Definition 1.1 Equation (1.1) is Ulam stable on $q^{\mathbb{N}_{0}}$ if there is a constant $C>0$ with the following property:

For any $\varepsilon>0$ and for any function $\zeta$ satisfying

$$
\mid D_{q} \zeta(t)-\alpha(t)\left\langle\left.\zeta(t)\right|_{\beta}\right| \leq \varepsilon \quad \text { for } t \in q^{\mathbb{N}_{0}}
$$

there is a solution $z$ of (1.1) such that

$$
|\zeta(t)-z(t)| \leq C \varepsilon \quad \text { for } t \in q^{\mathbb{N}_{0}}
$$

We call such $C$ a Ulam constant for (1.1) on $q^{\mathbb{N}_{0}}$.

The paper will proceed as follows. In the next section, we highlight the $q$-difference (quantum) exponential function and its properties and provide details on the solution to the related nonhomogeneous equation. In Sect. 3, we establish our main result, the Ulam stability of (1.1). In Sect. 4, we show some conditions under which (1.1) is Ulam unstable. 


\section{Exponential function and its properties}

In this section, we introduce the exponential function of equation (1.1). Define

$$
e_{\alpha}(t):=\prod_{j=0}^{\log _{q} t-1} \frac{1+(1-\beta)(q-1) q^{j} \alpha\left(q^{j}\right)}{1-\beta(q-1) q^{j} \alpha\left(q^{j}\right)}
$$

for $t \in q^{\mathbb{N} 0}$; note that for a function $f$, we define

$$
\prod_{j=0}^{-1} f(j):=1 \text {, }
$$

which is the standard definition. We immediately have the following lemma.

Lemma 2.1 Let $\alpha(t)$ satisfy (1.2), and let $e_{\alpha}(t)$ be given by (2.1). Then $e_{\alpha}(t)$ is the solution of $(1.1)$ with $e_{\alpha}(1)=1$. Moreover, $z(t)=z_{0} e_{\alpha}(t)$ is the solution of $(1.1)$ with $z(1)=z_{0}$, where $z_{0}$ is an arbitrary complex constant, that is, $z(t)=z_{0} e_{\alpha}(t)$ is the general solution of (1.1).

Proof It is clear that $e_{\alpha}(1)=1$. Now we will show that $e_{\alpha}(t)$ solves (1.1). Substituting it into the left side of (1.1) gives

$$
\begin{aligned}
D_{q} e_{\alpha}(t) & =\frac{1}{(q-1) t}\left[\frac{1+(1-\beta)(q-1) t \alpha(t)}{1-\beta(q-1) t \alpha(t)}-1\right] \prod_{j=0}^{\log _{q} t-1} \frac{1+(1-\beta)(q-1) q^{j} \alpha\left(q^{j}\right)}{1-\beta(q-1) q^{j} \alpha\left(q^{j}\right)} \\
& =\frac{\alpha(t)}{1-\beta(q-1) t \alpha(t)} e_{\alpha}(t) .
\end{aligned}
$$

On the other hand, substituting $e_{\alpha}(t)$ into the right side, we get

$$
\begin{aligned}
\alpha(t)\left\langle e_{\alpha}(t)\right\rangle_{\beta}= & \alpha(t)\left[\beta \frac{1+(1-\beta)(q-1) t \alpha(t)}{1-\beta(q-1) t \alpha(t)}+(1-\beta)\right] \\
& \times \prod_{j=0}^{\log _{q} t-1} \frac{1+(1-\beta)(q-1) q^{j} \alpha\left(q^{j}\right)}{1-\beta(q-1) q^{j} \alpha\left(q^{j}\right)} \\
= & \frac{\alpha(t)}{1-\beta(q-1) t \alpha(t)} e_{\alpha}(t) .
\end{aligned}
$$

Hence $e_{\alpha}(t)$ solves equation (1.1).

By $e_{\alpha}(1)=1$ we have $z(1)=z_{0}$. From the linearity of the solutions of linear equations we can conclude that $z(t)=z_{0} e_{\alpha}(t)$ is also a solution of (1.1). This completes the proof.

Needless to say, the function $e_{\alpha}(t)$ as defined above will play the role of the exponential function in $q$-difference equations.

Define

$$
\gamma(t):=\sum_{j=0}^{\log _{q} t-1} \frac{(q-1) q^{j} f\left(q^{j}\right)}{\left[1+(1-\beta)(q-1) q^{j} \alpha\left(q^{j}\right)\right] e_{\alpha}\left(q^{j}\right)} .
$$

The following lemma holds according to the method of variation of parameters. 
Lemma 2.2 Let $\alpha(t)$ satisfy (1.2), and let $e_{\alpha}(t)$ and $\gamma(t)$ be given by (2.1) and (2.2), respectively. Then the solution of the equation

$$
D_{q} \zeta(t)=\alpha(t)\left\langle\left.\zeta(t)\right|_{\beta}+f(t)\right.
$$

with $\zeta(1)=z_{0} \in \mathbb{C}$ is given by $\zeta(t)=\left(z_{0}+\gamma(t)\right) e_{\alpha}(t)$ for $t \in q^{\mathbb{N}_{0}}$, that is, $\zeta(t)=\left(z_{0}+\gamma(t)\right) e_{\alpha}(t)$ is the general solution of (2.3).

Proof Let $\zeta(t):=\eta(t) e_{\alpha}(t)$ for $t \in q^{\mathbb{N}_{0}}$, where $\eta(t)$ is an unclear function here. We assume that $\zeta(t)$ is a solution of (2.3). Noting that

$$
e_{\alpha}(q t)=\frac{1+(1-\beta)(q-1) t \alpha(t)}{1-\beta(q-1) t \alpha(t)} e_{\alpha}(t)
$$

we have

$$
\begin{aligned}
f(t)= & D_{q} \zeta(t)-\alpha(t)\langle\zeta(t)\rangle_{\beta}=D_{q}\left(\eta(t) e_{\alpha}(t)\right)-\alpha(t)\left\langle\left.\eta(t) e_{\alpha}(t)\right|_{\beta}\right. \\
= & \frac{\eta(q t) e_{\alpha}(q t)-\eta(t) e_{\alpha}(t)}{(q-1) t}-\alpha(t)\left[\beta \eta(q t) e_{\alpha}(q t)+(1-\beta) \eta(t) e_{\alpha}(t)\right] \\
= & {[1-\beta(q-1) t \alpha(t)] \frac{\eta(q t) e_{\alpha}(q t)}{(q-1) t}-[1+(1-\beta)(q-1) t \alpha(t)] \frac{\eta(t) e_{\alpha}(t)}{(q-1) t} } \\
= & \left\{[1-\beta(q-1) t \alpha(t)] \frac{1+(1-\beta)(q-1) t \alpha(t)}{1-\beta(q-1) t \alpha(t)} \eta(q t)\right. \\
& -[1+(1-\beta)(q-1) t \alpha(t)] \eta(t)\} \frac{e_{\alpha}(t)}{(q-1) t} \\
= & {[1+(1-\beta)(q-1) t \alpha(t)] e_{\alpha}(t) D_{q} \eta(t) . }
\end{aligned}
$$

This implies

$$
D_{q} \eta(t)=\frac{f(t)}{[1+(1-\beta)(q-1) t \alpha(t)] e_{\alpha}(t)}
$$

for $t \in q^{\mathbb{N}_{0}}$. Hence the solution of this equation is inductively obtained in the following form:

$$
\eta(t)=z_{0}+\sum_{j=0}^{\log _{q} t-1} \frac{(q-1) q^{j} f\left(q^{j}\right)}{\left[1+(1-\beta)(q-1) q^{j} \alpha\left(q^{j}\right)\right] e_{\alpha}\left(q^{j}\right)}
$$

for $t \in q^{\mathbb{N}_{0}}$.

Conversely, it satisfies the above equation. Indeed, we can check that

$$
\begin{aligned}
D_{q} \eta(t) & =\frac{\eta(q t)-\eta(t)}{(q-1) t}=\frac{1}{(q-1) t} \frac{(q-1) t f(t)}{[1+(1-\beta)(q-1) t \alpha(t)] e_{\alpha}(t)} \\
& =\frac{f(t)}{[1+(1-\beta)(q-1) t \alpha(t)] e_{\alpha}(t)}
\end{aligned}
$$


for $t \in q^{\mathbb{N}_{0}}$. If we go back to the above calculation, we can see that $\zeta(t)=\eta(t) e_{\alpha}(t)$ is the solution of (2.3) with $\zeta(1)=z_{0}$. Hence we have $\eta(t) \equiv z_{0}+\gamma(t)$, and this completes the proof.

Proposition 2.3 Let $\alpha(t)$ satisfy (1.2) and

$$
\liminf _{t \rightarrow \infty}|\alpha(t)|>0
$$

and let $e_{\alpha}(t)$ and $\gamma(t)$ be given by (2.1) and (2.2), respectively. If for any $\varepsilon>0$, the function $f(t)$ appearing in $\gamma(t)$ satisfies

$$
|f(t)| \leq \varepsilon \quad \text { for } t \in q^{\mathbb{N}_{0}}
$$

then:

(i) if $\beta \in\left[0, \frac{1}{2}\right)$, then $\lim _{t \rightarrow \infty}\left|e_{\alpha}(t)\right|=\infty, \lim _{t \rightarrow \infty} \gamma(t)$ exists, and

$$
\left|e_{\alpha}(t)\right| \sum_{j=\log _{q} t}^{\infty}\left|\frac{(q-1) q^{j}}{\left[1+(1-\beta)(q-1) q^{j} \alpha\left(q^{j}\right)\right] e_{\alpha}\left(q^{j}\right)}\right|
$$

is bounded above on $q^{\mathbb{N}_{0}}$;

(ii) if $\beta \in\left(\frac{1}{2}, 1\right]$, then $\lim _{t \rightarrow \infty}\left|e_{\alpha}(t)\right|=0$, and

$$
\left|e_{\alpha}(t)\right| \sum_{j=0}^{\log _{q} t-1}\left|\frac{(q-1) q^{j}}{\left[1+(1-\beta)(q-1) q^{j} \alpha\left(q^{j}\right)\right] e_{\alpha}\left(q^{j}\right)}\right|
$$

is bounded above on $q^{\mathbb{N}_{0}}$.

Proof By (2.4) we have $\lim _{t \rightarrow \infty} t|\alpha(t)|=\infty$. It follows that

$$
\begin{aligned}
\lim _{j \rightarrow \infty}\left|\frac{1+(1-\beta)(q-1) q^{j} \alpha\left(q^{j}\right)}{1-\beta(q-1) q^{j} \alpha\left(q^{j}\right)}\right| & =\lim _{j \rightarrow \infty}\left|\frac{\frac{1}{q^{j}\left|\alpha\left(q^{j}\right)\right|}+(1-\beta)(q-1) \frac{\alpha\left(q^{j}\right)}{\left|\alpha\left(q^{j}\right)\right|}}{\frac{1}{q^{j}\left|\alpha\left(q^{j}\right)\right|}-\beta(q-1) \frac{\alpha\left(q^{j}\right)}{\left|\alpha\left(q^{j}\right)\right|}}\right| \\
& = \begin{cases}\infty, & \beta=0, \\
\frac{1-\beta}{\beta} \in(1, \infty), & \beta \in\left(0, \frac{1}{2}\right), \\
1: & \beta=\frac{1}{2}, \\
\frac{1-\beta}{\beta} \in(0,1), & \beta \in\left(\frac{1}{2}, 1\right), \\
0, & \beta=1 .\end{cases}
\end{aligned}
$$

Let

$$
\underline{\alpha}:=\liminf _{t \rightarrow \infty}|\alpha(t)|>0
$$

Then we obtain

$$
\limsup _{j \rightarrow \infty}\left|\frac{(q-1) q^{j}}{1+(1-\beta)(q-1) q^{j} \alpha\left(q^{j}\right)}\right|=\limsup _{j \rightarrow \infty}\left|\frac{q-1}{\frac{1}{q^{j}}+(1-\beta)(q-1) \alpha\left(q^{j}\right)}\right|=\frac{1}{(1-\beta) \underline{\alpha}}
$$


so there is a constant $L>0$ such that

$$
\left|\frac{(q-1) q^{j}}{1+(1-\beta)(q-1) q^{j} \alpha\left(q^{j}\right)}\right| \leq L
$$

for $j \in q^{\mathbb{N}_{0}}$.

First, we consider case (i). Let $\beta \in\left[0, \frac{1}{2}\right)$. By (2.6) there exist constants $\mu_{1}>1$ and $k \in \mathbb{N}$ such that

$$
\left|\frac{1+(1-\beta)(q-1) q^{j} \alpha\left(q^{j}\right)}{1-\beta(q-1) q^{j} \alpha\left(q^{j}\right)}\right| \geq \mu_{1}>1
$$

for $j \geq k$, and thus

$$
\left|e_{\alpha}(t)\right| \geq\left[\prod_{j=0}^{k-1}\left|\frac{1+(1-\beta)(q-1) q^{j} \alpha\left(q^{j}\right)}{1-\beta(q-1) q^{j} \alpha\left(q^{j}\right)}\right|\right]\left[\prod_{j=k}^{\log _{q} t-1} \mu_{1}\right]=v_{1} \mu_{1}^{\log _{q} t-k}
$$

for $t \geq q^{k}$, where

$$
v_{1}:=\prod_{j=0}^{k-1}\left|\frac{1+(1-\beta)(q-1) q^{j} \alpha\left(q^{j}\right)}{1-\beta(q-1) q^{j} \alpha\left(q^{j}\right)}\right|
$$

This implies that $\lim _{t \rightarrow \infty}\left|e_{\alpha}(t)\right|=\infty$.

Next, we will show that $\lim _{t \rightarrow \infty} \gamma(t)$ exists. Using (2.5), (2.7), and (2.9), we have

$$
\begin{aligned}
\lim _{t \rightarrow \infty}|\gamma(t)| & \leq \lim _{t \rightarrow \infty} \sum_{j=0}^{\log _{q} t-1}\left|\frac{(q-1) q^{j} f\left(q^{j}\right)}{\left[1+(1-\beta)(q-1) q^{j} \alpha\left(q^{j}\right)\right] e_{\alpha}\left(q^{j}\right)}\right| \leq \varepsilon L \lim _{t \rightarrow \infty} \sum_{j=0}^{\log _{q} t-1} \frac{1}{\left|e_{\alpha}\left(q^{j}\right)\right|} \\
& \leq \varepsilon L\left[\sum_{j=0}^{k-1} \frac{1}{\left|e_{\alpha}\left(q^{j}\right)\right|}+\frac{1}{v_{1}} \lim _{t \rightarrow \infty} \sum_{j=k}^{\log _{q} t-1}\left(\frac{1}{\mu_{1}}\right)^{j-k}\right] \\
& =\varepsilon L\left[\sum_{j=0}^{k-1} \frac{1}{\left|e_{\alpha}\left(q^{j}\right)\right|}+\frac{\mu_{1}}{v_{1}\left(\mu_{1}-1\right)}\right]<\infty .
\end{aligned}
$$

Consequently,

$$
\lim _{t \rightarrow \infty} \gamma(t)=\sum_{j=0}^{\infty} \frac{(q-1) q^{j} f\left(q^{j}\right)}{\left[1+(1-\beta)(q-1) q^{j} \alpha\left(q^{j}\right)\right] e_{\alpha}\left(q^{j}\right)}
$$

exists.

By (2.7) and (2.8) we obtain

$$
\begin{aligned}
& \left|e_{\alpha}(t)\right| \sum_{j=\log _{q} t}^{\infty}\left|\frac{(q-1) q^{j}}{\left[1+(1-\beta)(q-1) q^{j} \alpha\left(q^{j}\right)\right] e_{\alpha}\left(q^{j}\right)}\right| \\
& \quad \leq L\left|e_{\alpha}(t)\right| \sum_{j=\log _{q} t}^{\infty} \frac{1}{\left|e_{\alpha}\left(q^{j}\right)\right|}=L\left|e_{\alpha}(t)\right|\left(\frac{1}{\left|e_{\alpha}(t)\right|}+\frac{1}{\left|e_{\alpha}(q t)\right|}+\frac{1}{\left|e_{\alpha}\left(q^{2} t\right)\right|}+\cdots\right)
\end{aligned}
$$




$$
\begin{aligned}
= & L\left(1+\left|\frac{1-\beta(q-1) t \alpha(t)}{1+(1-\beta)(q-1) t \alpha(t)}\right|\right. \\
& \left.+\left|\frac{1-\beta(q-1) t \alpha(t)}{1+(1-\beta)(q-1) t \alpha(t)}\right|\left|\frac{1-\beta(q-1) q t \alpha(q t)}{1+(1-\beta)(q-1) q t \alpha(q t)}\right|+\cdots\right) \\
= & L \sum_{j=0}^{\infty}\left(\prod_{m=0}^{j-1}\left|\frac{1-\beta(q-1) q^{m} t \alpha\left(q^{m} t\right)}{1+(1-\beta)(q-1) q^{m} t \alpha\left(q^{m} t\right)}\right|\right) \\
\leq L & \sum_{j=0}^{\infty}\left[\prod_{m=0}^{j-1}\left(\frac{1}{\mu_{1}}\right)\right]=L \sum_{j=0}^{\infty}\left(\frac{1}{\mu_{1}}\right)^{j}=\frac{L \mu_{1}}{\mu_{1}-1}<\infty
\end{aligned}
$$

for $t \geq q^{k}$.

Next, we consider case (ii). Let $\beta \in\left(\frac{1}{2}, 1\right]$. By (2.6) there exist constants $0<\mu_{2}<1$ and $l \in \mathbb{N}$ such that

$$
\left|\frac{1+(1-\beta)(q-1) q^{j} \alpha\left(q^{j}\right)}{1-\beta(q-1) q^{j} \alpha\left(q^{j}\right)}\right| \leq \mu_{2}<1
$$

for $j \geq l$, and thus

$$
\begin{aligned}
& \prod_{m=j}^{\log _{q} t-1}\left|\frac{1+(1-\beta)(q-1) q^{m} \alpha\left(q^{m}\right)}{1-\beta(q-1) q^{m} \alpha\left(q^{m}\right)}\right| \\
& \leq\left\lceil\prod_{m=j}^{l-1}\left|\frac{1+(1-\beta)(q-1) q^{m} \alpha\left(q^{m}\right)}{1-\beta(q-1) q^{m} \alpha\left(q^{m}\right)}\right|\right]\left[\prod_{m=l}^{\log _{q} t-1} \mu_{2}\right] \\
& \leq v_{2} \mu_{2}^{\log _{q} t-l}
\end{aligned}
$$

for $0 \leq j \leq l-1$ and $t \geq q^{l+1}$, where

$$
v_{2}:=\max _{0 \leq j \leq l-1}\left\{\prod_{m=j}^{l-1}\left|\frac{1+(1-\beta)(q-1) q^{m} \alpha\left(q^{m}\right)}{1-\beta(q-1) q^{m} \alpha\left(q^{m}\right)}\right|\right\} .
$$

This, with $j=0$, implies that $\lim _{t \rightarrow \infty}\left|e_{\alpha}(t)\right|=0$.

By (2.7) we have

$$
\begin{aligned}
& \left|e_{\alpha}(t)\right| \sum_{j=0}^{\log _{q} t-1}\left|\frac{(q-1) q^{j}}{\left[1+(1-\beta)(q-1) q^{j} \alpha\left(q^{j}\right)\right] e_{\alpha}\left(q^{j}\right)}\right| \\
& \leq L\left|e_{\alpha}(t)\right| \sum_{j=0}^{\log _{q} t-1} \frac{1}{\left|e_{\alpha}\left(q^{j}\right)\right|}=L\left|e_{\alpha}(t)\right|\left(\frac{1}{\left|e_{\alpha}(1)\right|}+\frac{1}{\left|e_{\alpha}(q)\right|}+\frac{1}{\left|e_{\alpha}\left(q^{2}\right)\right|}+\cdots+\frac{1}{\left|e_{\alpha}\left(q^{-1} t\right)\right|}\right) \\
& =L\left(\prod_{j=1}^{\log _{q} t-1}\left|\frac{1+(1-\beta)(q-1) q^{j} \alpha\left(q^{j}\right)}{1-\beta(q-1) q^{j} \alpha\left(q^{j}\right)}\right|+\prod_{j=2}^{\log _{q} t-1}\left|\frac{1+(1-\beta)(q-1) q^{j} \alpha\left(q^{j}\right)}{1-\beta(q-1) q^{j} \alpha\left(q^{j}\right)}\right|\right. \\
& \left.\quad+\cdots+\prod_{j=\log _{q} t-2}\left|\frac{1+(1-\beta)(q-1) q^{j} \alpha\left(q^{j}\right)}{1-\beta(q-1) q^{j} \alpha\left(q^{j}\right)}\right|\right)
\end{aligned}
$$




$$
\begin{aligned}
= & L \sum_{j=1}^{\log _{q} t-2}\left(\prod_{m=j}^{\log _{q} t-1}\left|\frac{1+(1-\beta)(q-1) q^{m} \alpha\left(q^{m}\right)}{1-\beta(q-1) q^{m} \alpha\left(q^{m}\right)}\right|\right) \\
= & L \sum_{j=1}^{l-1}\left(\prod_{m=j}^{\log _{q} t-1}\left|\frac{1+(1-\beta)(q-1) q^{m} \alpha\left(q^{m}\right)}{1-\beta(q-1) q^{m} \alpha\left(q^{m}\right)}\right|\right) \\
& +L \sum_{j=l}^{\log _{q} t-2}\left(\prod_{m=j}^{\log _{q} t-1}\left|\frac{1+(1-\beta)(q-1) q^{m} \alpha\left(q^{m}\right)}{1-\beta(q-1) q^{m} \alpha\left(q^{m}\right)}\right|\right) .
\end{aligned}
$$

Moreover, using (2.10) and (2.11), we obtain

$$
\begin{aligned}
& \left|e_{\alpha}(t)\right| \sum_{j=0}^{\log _{q} t-1}\left|\frac{(q-1) q^{j}}{\left[1+(1-\beta)(q-1) q^{j} \alpha\left(q^{j}\right)\right] e_{\alpha}\left(q^{j}\right)}\right| \\
& \leq L \sum_{j=1}^{l-1} v_{2} \mu_{2}^{\log _{q} t-l}+L \sum_{j=l}^{\log _{q} t-2}\left(\prod_{m=j}^{\log _{q} t-1} \mu_{2}\right) \\
& =L(l-1) \nu_{2} \mu_{2}^{\log _{q} t-l}+L \sum_{j=l}^{\log _{q} t-2} \mu_{2}^{\log _{q} t-j} \\
& =L(l-1) \nu_{2} \mu_{2}^{\log _{q} t-l}+L \frac{\mu_{2}\left(\mu_{2}-\mu_{2}^{\log _{q} t-l}\right)}{1-\mu_{2}}<L v_{2}(l-1)+L \frac{\mu_{2}^{2}}{1-\mu_{2}}<\infty
\end{aligned}
$$

for $t \geq q^{l+1}$. This completes the proof.

\section{Ulam stability}

The main Ulam stability result of this paper is as follows.

Theorem 3.1 Let $\alpha(t)$ satisfy (1.2) and (2.4), and let $e_{\alpha}(t)$ be given by (2.1). Let $\varepsilon>0$ be arbitrary. Suppose that $\zeta(t)$ satisfies (2.3) with (2.5). Then:

(i) if $\beta \in\left[0, \frac{1}{2}\right)$, then $\lim _{t \rightarrow \infty} \frac{\zeta(t)}{e_{\alpha}(t)}$ exists, the function

$$
z_{1}(t):=\left(\lim _{t \rightarrow \infty} \frac{\zeta(t)}{e_{\alpha}(t)}\right) e_{\alpha}(t)
$$

uniquely fulfills (1.1), and $\left|\zeta(t)-z_{1}(t)\right| \leq C_{1} \varepsilon$ for all $t \in q^{\mathbb{N}_{0}}$, where

$$
C_{1}:=\sup _{t \in q^{\mathbb{N} 0}}\left|e_{\alpha}(t)\right| \sum_{j=\log _{q} t}^{\infty}\left|\frac{(q-1) q^{j}}{\left[1+(1-\beta)(q-1) q^{j} \alpha\left(q^{j}\right)\right] e_{\alpha}\left(q^{j}\right)}\right|<\infty ;
$$

(ii) if $\beta \in\left(\frac{1}{2}, 1\right]$, then there is a constant $z_{0} \in \mathbb{C}$ such that

$$
z_{2}(t):=z_{0} e_{\alpha}(t)
$$

fulfills (1.1), and $\left|\zeta(t)-z_{2}(t)\right| \leq C_{2} \varepsilon$ for all $t \in q^{\mathbb{N}_{0}}$, where

$$
C_{2}:=\sup _{t \in q^{\mathbb{N} 0}}\left|e_{\alpha}(t)\right| \sum_{j=0}^{\log _{q} t-1}\left|\frac{(q-1) q^{j}}{\left[1+(1-\beta)(q-1) q^{j} \alpha\left(q^{j}\right)\right] e_{\alpha}\left(q^{j}\right)}\right|<\infty .
$$


Proof Let $\varepsilon>0$. We suppose that $\alpha(t)$ satisfies (1.2) and (2.4), whereas $\zeta(t)$ satisfies (2.3) with (2.5). By Lemma 2.2 we can write $\zeta(t)$ in the form

$$
\zeta(t)=\left(z_{0}+\gamma(t)\right) e_{\alpha}(t)
$$

for some $z_{0} \in \mathbb{C}$, where $\gamma(t)$ is given by (2.2).

First, we consider case (i), that is, suppose $\beta \in\left[0, \frac{1}{2}\right)$. From Proposition 2.3 we see that

$$
\lim _{t \rightarrow \infty} \frac{\zeta(t)}{e_{\alpha}(t)}=z_{0}+\lim _{t \rightarrow \infty} \gamma(t)
$$

exists. Using this, define the function

$$
z_{1}(t):=\left(\lim _{t \rightarrow \infty} \frac{\zeta(t)}{e_{\alpha}(t)}\right) e_{\alpha}(t)
$$

for $t \in q^{\mathbb{N}_{0}}$. Then from Lemma 2.1 we note that $z_{1}(t)$ is the solution of (1.1) with $z_{1}(1)=$ $\left(\lim _{t \rightarrow \infty} \frac{\zeta(t)}{e_{\alpha}(t)}\right)$. Hence by Proposition 2.3 and $(2.5)$ we obtain

$$
\begin{aligned}
\left|\zeta(t)-z_{1}(t)\right| & =\left|\left(z_{0}+\gamma(t)\right) e_{\alpha}(t)-\left(z_{0}+\lim _{t \rightarrow \infty} \gamma(t)\right) e_{\alpha}(t)\right| \\
& =\left|\left(\gamma(t)-\lim _{t \rightarrow \infty} \gamma(t)\right) e_{\alpha}(t)\right| \\
& =\left|e_{\alpha}(t) \sum_{j=\log _{q} t}^{\infty} \frac{(q-1) q^{j} f\left(q^{j}\right)}{\left[1+(1-\beta)(q-1) q^{j} \alpha\left(q^{j}\right)\right] e_{\alpha}\left(q^{j}\right)}\right| \\
& \leq \varepsilon\left|e_{\alpha}(t)\right| \sum_{j=\log _{q} t}^{\infty}\left|\frac{(q-1) q^{j}}{\left[1+(1-\beta)(q-1) q^{j} \alpha\left(q^{j}\right)\right] e_{\alpha}\left(q^{j}\right)}\right| \\
& \leq \varepsilon \sup _{t \in q^{\mathbb{N} 0}}\left|e_{\alpha}(t)\right| \sum_{j=\log _{q} t}^{\infty}\left|\frac{(q-1) q^{j}}{\left[1+(1-\beta)(q-1) q^{j} \alpha\left(q^{j}\right)\right] e_{\alpha}\left(q^{j}\right)}\right|<\infty
\end{aligned}
$$

for $t \in q^{\mathbb{N}_{0}}$.

Next, we will show that $z_{1}(t)$ satisfies (1.1) and $\left|\zeta(t)-z_{1}(t)\right| \leq C_{1} \varepsilon$ uniquely. Consider the function

$$
y(t):=y_{0} e_{\alpha}(t)
$$

satisfying $|\zeta(t)-y(t)| \leq C_{1} \varepsilon$ for $t \in q^{\mathbb{N}_{0}}$, where $y_{0} \neq\left(\lim _{t \rightarrow \infty} \frac{\zeta(t)}{e_{\alpha}(t)}\right)$. From Lemma 2.1 it follows that $y(t)$ satisfies (1.1). Hence we obtain

$$
\left|\left(\lim _{t \rightarrow \infty} \frac{\zeta(t)}{e_{\alpha}(t)}\right)-y_{0}\right|\left|e_{\alpha}(t)\right|=\left|z_{1}(t)-y(t)\right| \leq\left|\zeta(t)-z_{1}(t)\right|+|\zeta(t)-y(t)| \leq 2 C_{1} \varepsilon
$$

for $t \in q^{\mathbb{N}_{0}}$. By Proposition 2.3 we know that $\lim _{t \rightarrow \infty}\left|e_{\alpha}(t)\right|=\infty$, and so the above inequality derives a contradiction.

Next, we consider case (ii), that is, suppose $\beta \in\left(\frac{1}{2}, 1\right]$. Let

$$
z_{2}(t):=z_{0} e_{\alpha}(t)
$$


Then by Lemma $2.1 z_{2}(t)$ is a solution of (1.1). Using Proposition 2.3, we see that

$$
\begin{aligned}
\left|\zeta(t)-z_{2}(t)\right| & =\left|\left(z_{0}+\gamma(t)\right) e_{\alpha}(t)-z_{0} e_{\alpha}(t)\right|=\left|\gamma(t) e_{\alpha}(t)\right| \\
& =\left|e_{\alpha}(t) \sum_{j=0}^{\log _{q} t-1} \frac{(q-1) q^{j} f\left(q^{j}\right)}{\left[1+(1-\beta)(q-1) q^{j} \alpha\left(q^{j}\right)\right] e_{\alpha}\left(q^{j}\right)}\right| \\
& \leq \varepsilon\left|e_{\alpha}(t)\right| \sum_{j=0}^{\log _{q} t-1}\left|\frac{(q-1) q^{j}}{\left[1+(1-\beta)(q-1) q^{j} \alpha\left(q^{j}\right)\right] e_{\alpha}\left(q^{j}\right)}\right| \\
& \leq \varepsilon \sup _{t \in q^{\mathbb{N}} 0}\left|e_{\alpha}(t)\right| \sum_{j=0}^{\log _{q} t-1}\left|\frac{(q-1) q^{j}}{\left[1+(1-\beta)(q-1) q^{j} \alpha\left(q^{j}\right)\right] e_{\alpha}\left(q^{j}\right)}\right|<\infty
\end{aligned}
$$

for $t \in q^{\mathbb{N}_{0}}$. Consequently, the statement in this theorem is true. This completes the proof.

Remark 3.2 The results in Theorem 3.1 include and extend the results given in [8, Theorem 2.6] and [9, Theorem 2.4], which deal with the Ulam stability when the coefficient is a complex constant. Let

$$
g(j):=\frac{(q-1) q^{j}}{\left[1+(1-\beta)(q-1) q^{j} \alpha\left(q^{j}\right)\right] e_{\alpha}\left(q^{j}\right)}
$$

for $j \in \mathbb{N}_{0}$. According to the results in [8, Theorem 2.8] and [9, Theorem 2.6], the following facts hold under the assumption that $\alpha(t)$ satisfies (1.2) and $\alpha(t) \equiv \alpha \neq 0$ :

(i) if $\beta \in\left[0, \frac{1}{2}\right)$ and $\sum_{j=\log _{q} t}^{\infty}|g(j)|=\left|\sum_{j=\log _{q} t}^{\infty} g(j)\right|$, then (1.1) is Ulam stable with the best Ulam constant $C_{1}=\frac{1}{|\alpha|}$.

(ii) if $\beta \in\left(\frac{1}{2}, 1\right]$ and $\sum_{j=0}^{\log _{q} t-1}|g(j)|=\left|\sum_{j=0}^{\log _{q} t-1} g(j)\right|$ for sufficiently large $t \in q^{\mathbb{N}_{0}}$, then (1.1) is Ulam stable, and there is $\delta>0$ such that $C_{2}=\frac{1}{|\alpha|}+\delta$ is an Ulam constant for sufficiently large $t \in q^{\mathbb{N}_{0}}$.

A natural follow-up question is what happens if $\beta=\frac{1}{2}$ ? We give partial answers in the following theorem and in the next section on instability.

Theorem 3.3 Let $q>1$ and $\beta=\frac{1}{2}$, and let $\alpha(t)$ satisfy (1.2). If $\alpha(t)$ also satisfies

$$
\lim _{t \rightarrow \infty}\left|\frac{\alpha(t)}{\alpha(q t)}\right|<1
$$

then (1.1) is Ulam stable on $q^{\mathbb{N}_{0}}$.

Proof In addition to the hypotheses in the statement of this theorem, let $e_{\alpha}(t)$ be given by (2.1). Note that (3.2) implies (2.4). With $\beta=\frac{1}{2}$, the exponential function takes the form

$$
e_{\alpha}(t)=\prod_{j=0}^{\log _{q} t-1} \frac{1+\frac{1}{2}(q-1) q^{j} \alpha\left(q^{j}\right)}{1-\frac{1}{2}(q-1) q^{j} \alpha\left(q^{j}\right)}, \quad t \in q^{\mathbb{N}_{0}},
$$


and

$$
\lim _{t \rightarrow \infty} \frac{1+\frac{1}{2}(q-1) q^{j} \alpha\left(q^{j}\right)}{1-\frac{1}{2}(q-1) q^{j} \alpha\left(q^{j}\right)}=-1
$$

since $\alpha$ satisfies conditions (1.2) and (2.4). It follows that $e_{\alpha}(t)$ converges to a two-cycle $\pm \xi^{*}$ for some $\xi^{*} \in \mathbb{C} \backslash\{0\}$ as $t \rightarrow \infty$. Let $\varepsilon>0$ be arbitrary. For $\gamma(t)$ given by (2.2) with $(2.5)$,

$$
|\gamma(t)| \leq \varepsilon \sum_{j=0}^{\log _{q} t-1} \frac{(q-1) q^{j}}{\left|1+\frac{1}{2}(q-1) q^{j} \alpha\left(q^{j}\right)\right| \cdot\left|e_{\alpha}\left(q^{j}\right)\right|}
$$

Using the ratio test, we have

$$
\begin{aligned}
\lim _{j \rightarrow \infty} \frac{\left|q-\frac{1}{2}(q-1) q^{j+1} \alpha\left(q^{j}\right)\right|}{\left|1+\frac{1}{2}(q-1) q^{j+1} \alpha\left(q^{j+1}\right)\right|} & =\lim _{j \rightarrow \infty}\left|\frac{\frac{q}{\frac{1}{2}(q-1) q^{j+1}\left|\alpha\left(q^{j}\right)\right|}-\frac{\alpha\left(q^{j}\right)}{\left|\alpha\left(q^{j}\right)\right|}}{\frac{1}{\frac{1}{2}(q-1) q^{j+1}\left|\alpha\left(q^{j+1}\right)\right|}+\frac{\alpha\left(q^{j+1}\right)}{\left|\alpha\left(q^{j+1}\right)\right|}}\right|\left|\frac{\alpha\left(q^{j}\right)}{\alpha\left(q^{j+1}\right)}\right| \\
& =\lim _{j \rightarrow \infty}\left|\frac{\alpha\left(q^{j}\right)}{\alpha\left(q^{j+1}\right)}\right|<1
\end{aligned}
$$

by assumption, so that $\gamma(t)$ converges absolutely. Suppose that $\zeta(t)$ satisfies (2.3) with (2.5), and suppose that $z(t)$ satisfies (1.1) with $z(1)=z_{0}$. Then

$$
|\zeta(t)-z(t)|=\left|\left(z_{0}+\gamma(t)\right) e_{\alpha}(t)-z_{0} e_{\alpha}(t)\right|=\left|\gamma(t) e_{\alpha}(t)\right| \leq C_{3} \varepsilon
$$

where

$$
C_{3}:=\sup _{t \in q^{\mathbb{N}} 0}\left|e_{\alpha}(t)\right| \sum_{j=0}^{\log _{q} t-1}\left|\frac{(q-1) q^{j}}{\left[1+\frac{1}{2}(q-1) q^{j} \alpha\left(q^{j}\right)\right] e_{\alpha}\left(q^{j}\right)}\right|<\infty .
$$

This completes the proof.

Remark 3.4 Note that $C_{2}$ in Theorem 3.1(ii) and $C_{3}$ given in (3.3) are the same when $\beta=\frac{1}{2}$.

The theorems in this section can be summarized as follows.

Theorem 3.5 If $\alpha(t)$ satisfies (1.2) and (2.4) for $\beta \in\left[0, \frac{1}{2}\right) \cup\left(\frac{1}{2}, 1\right]$, then (1.1) is Ulam stable on $q^{\mathbb{N}_{0}}$. If $\alpha(t)$ satisfies (1.2), (2.4), and (3.2) for $\beta=\frac{1}{2}$, then (1.1) is Ulam stable on $q^{\mathbb{N}_{0}}$.

\section{Ulam instability}

What happens if the coefficient function $\alpha$ fails to satisfy (2.4)? In the following example, we show an example where (1.1) is unstable in the Ulam sense.

Example 4.1 Let $q>1$ be fixed, take $\rho \in(-\infty,-1) \cup(1, \infty)$, and let

$$
\alpha(t)=\frac{1}{(q-1) \rho t}, \quad t \in q^{\mathbb{N}_{0}}
$$


We easily see that this $\alpha$ satisfies (1.2) for any $\beta \in[0,1]$ but fails to satisfy (2.4). Plugging this $\alpha$ into (2.1), we have the exponential function

$$
e_{\alpha}(t)=\prod_{j=0}^{\log _{q} t-1} \frac{1+\frac{1}{\rho}(1-\beta)}{1-\frac{1}{\rho} \beta}=\left(\frac{1+\rho-\beta}{\rho-\beta}\right)^{\log _{q} t} \text {. }
$$

For arbitrary $\varepsilon>0$, let $f(t) \equiv \varepsilon$. Substituting these $\alpha, e_{\alpha}$, and $f$ into (2.2), we have

$$
\gamma(t)=\sum_{j=0}^{\log _{q} t-1} \frac{(q-1) q^{j} f\left(q^{j}\right)}{\left[1+\frac{1}{\rho}(1-\beta)\right] e_{\alpha}\left(q^{j}\right)}=\frac{(q-1) \rho \varepsilon}{1+\rho-\beta} \sum_{j=0}^{\log _{q} t-1}\left(\frac{(\rho-\beta) q}{1+\rho-\beta}\right)^{j} \geq 0 .
$$

It follows that $\zeta(t)=\gamma(t) e_{\alpha}(t)$ is a solution of (2.3). Let $z(t)=z_{0} e_{\alpha}(t)$ be any solution of (1.1). There are three cases.

Case i. Let $\beta \in[0,1]$ and $\rho \in(-\infty,-1)$. Clearly, $e_{\alpha}(t)$ in (4.1) goes to zero as $t$ goes to infinity in $q^{\mathbb{N}_{0}}$. Moreover, $\gamma(t)$ in $(4.2)$ and $\zeta(t)=\gamma(t) e_{\alpha}(t)$ diverge to positive infinity as $t$ goes to positive infinity, so that

$$
|\zeta(t)-z(t)|=\left|\gamma(t) e_{\alpha}(t)-z_{0} e_{\alpha}(t)\right| \rightarrow \infty
$$

for any $z_{0} \in \mathbb{C}$, and (1.1) is not Ulam stable.

Case ii. Let $\beta \in[0,1]$ and $\rho \in(1, \infty)$. Clearly, $e_{\alpha}(t)$ in (4.1) goes to infinity as $t$ goes to infinity in $q^{\mathbb{N}_{0}}$. Suppose $q \geq \frac{1+\rho-\beta}{\rho-\beta}$. Then $\gamma(t)$ in (4.2) diverges to positive infinity as $t$ goes to positive infinity, so that

$$
|\zeta(t)-z(t)|=\left|\gamma(t)-z_{0}\right| e_{\alpha}(t) \rightarrow \infty
$$

for any $z_{0} \in \mathbb{C}$, and (1.1) is not Ulam stable.

Case iii. Let $\beta \in[0,1]$ and $\rho \in(1, \infty)$. As in (ii), $e_{\alpha}(t)$ in (4.1) goes to infinity as $t$ goes to infinity. Suppose $1<q<\frac{1+\rho-\beta}{\rho-\beta}$. Then $\gamma$ in (4.2) is a convergent geometric series. Using this fact, rewrite (4.2) as

$$
\begin{aligned}
\gamma(t) & =\frac{(q-1) \rho \varepsilon}{1+\rho-\beta} \sum_{j=0}^{\infty}\left(\frac{(\rho-\beta) q}{1+\rho-\beta}\right)^{j}-\frac{(q-1) \rho \varepsilon}{1+\rho-\beta} \sum_{j=\log _{q} t}^{\infty}\left(\frac{(\rho-\beta) q}{1+\rho-\beta}\right)^{j} \\
& =\frac{(q-1) \rho \varepsilon}{(1+\rho-\beta)-q(\rho-\beta)}-\frac{(q-1) \rho \varepsilon t\left(\frac{\rho-\beta}{1+\rho-\beta}\right)^{\log _{q} t}}{(1+\rho-\beta)-q(\rho-\beta)} .
\end{aligned}
$$

Consequently,

$$
\begin{aligned}
|\zeta(t)-z(t)|= & \left|\gamma(t)-z_{0}\right| e_{\alpha}(t) \\
= & \left|\frac{(q-1) \rho \varepsilon}{(1+\rho-\beta)-q(\rho-\beta)}-\frac{(q-1) \rho \varepsilon t\left(\frac{\rho-\beta}{1+\rho-\beta}\right)^{\log _{q} t}}{(1+\rho-\beta)-q(\rho-\beta)}-z_{0}\right| \\
& \times\left(\frac{1+\rho-\beta}{\rho-\beta}\right)^{\log _{q} t} .
\end{aligned}
$$


If $z_{0}=0$, then

$$
\begin{aligned}
& \lim _{t \rightarrow \infty}|\zeta(t)-z(t)| \\
& =\lim _{t \rightarrow \infty}\left|\frac{(q-1) \rho \varepsilon}{(1+\rho-\beta)-q(\rho-\beta)}-\frac{(q-1) \rho \varepsilon t\left(\frac{\rho-\beta}{1+\rho-\beta}\right)^{\log _{q} t}}{(1+\rho-\beta)-q(\rho-\beta)}\right|\left(\frac{1+\rho-\beta}{\rho-\beta}\right)^{\log _{q} t} \\
& =\frac{(q-1) \rho \varepsilon}{(1+\rho-\beta)-q(\rho-\beta)} \lim _{t \rightarrow \infty}\left(\frac{1+\rho-\beta}{\rho-\beta}\right)^{\log _{q} t}=\infty,
\end{aligned}
$$

and (1.1) is not Ulam stable. If $z_{0}=\frac{(q-1) \rho \varepsilon}{(1+\rho-\beta)-q(\rho-\beta)}$, then

$$
\begin{aligned}
\lim _{t \rightarrow \infty}|\zeta(t)-z(t)| & =\lim _{t \rightarrow \infty}\left|-\frac{(q-1) \rho \varepsilon t\left(\frac{\rho-\beta}{1+\rho-\beta}\right)^{\log _{q} t}}{(1+\rho-\beta)-q(\rho-\beta)}\right|\left(\frac{1+\rho-\beta}{\rho-\beta}\right)^{\log _{q} t} \\
& =\lim _{t \rightarrow \infty} \frac{\rho \varepsilon(q-1) t}{(1+\rho-\beta)-q(\rho-\beta)}=\infty,
\end{aligned}
$$

and again (1.1) is not Ulam stable. Any other choice of $z_{0} \in \mathbb{C}$ leads to a similar conclusion. Therefore (1.1) is Ulam unstable in all cases for this example.

Theorem 4.2 Let $\alpha(t)$ satisfy (1.2) and

$$
\limsup _{t \rightarrow \infty}|\alpha(t)|<\infty
$$

and let $e_{\alpha}(t)$ be given by (2.1). If

$$
0<\liminf _{t \rightarrow \infty}\left|e_{\alpha}(t)\right| \text { and } \limsup _{t \rightarrow \infty}\left|e_{\alpha}(t)\right|<\infty \text {, }
$$

then (1.1) is unstable in the Ulam sense.

Proof For arbitrary $\varepsilon>0$, let

$$
f(t) \equiv \frac{\varepsilon\left[1+(1-\beta)(q-1) q^{j} \alpha\left(q^{j}\right)\right] e_{\alpha}(t)}{\left|\left[1+\beta(q-1) q^{j} \alpha\left(q^{j}\right)\right] e_{\alpha}(t)\right|} .
$$

Substituting this $f$ into (2.2), we have

$$
\gamma(t)=\varepsilon \sum_{j=0}^{\log _{q} t-1} \frac{(q-1) q^{j}}{\left|1+\beta(q-1) q^{j} \alpha\left(q^{j}\right)\right|\left|e_{\alpha}\left(q^{j}\right)\right|} .
$$

From Lemma 2.2 it follows that $\zeta(t)=\gamma(t) e_{\alpha}(t)$ is a solution of (2.3). Let $z(t)=z_{0} e_{\alpha}(t)$ be any solution of (1.1).

From conditions (4.3) and (4.4) we see that there exists a constant $\mu_{1}>0$ such that

$$
\frac{(q-1) q^{j}}{\left|1+\beta(q-1) q^{j} \alpha\left(q^{j}\right)\right|}=\frac{1}{\left|\frac{1}{(q-1) q^{j}}+\beta \alpha\left(q^{j}\right)\right|} \geq \mu_{1}
$$


for all $j \in \mathbb{N}_{0}$, and there exist $\mu_{2}, \mu_{3}>0$ and $v \in \mathbb{N}_{0}$ such that

$$
\mu_{2} \leq\left|e_{\alpha}(t)\right| \leq \mu_{3}
$$

for all $t \geq q^{v}$. This, together with (4.5), yields

$$
\begin{aligned}
\gamma(t) & =\varepsilon \sum_{j=0}^{\log _{q} t-1} \frac{1}{\left|\frac{1}{(q-1) q^{j}}+\beta \alpha\left(q^{j}\right)\right|\left|e_{\alpha}\left(q^{j}\right)\right|} \geq \varepsilon \mu_{1} \sum_{j=0}^{\log _{q} t-1} \frac{1}{\left|e_{\alpha}\left(q^{j}\right)\right|} \\
& \geq \varepsilon \mu_{1}\left(\sum_{j=0}^{v-1} \frac{1}{\left|e_{\alpha}\left(q^{j}\right)\right|}+\frac{1}{\mu_{3}} \sum_{j=v}^{\log _{q} t-1} 1\right) \\
& =\varepsilon \mu_{1}\left(\sum_{j=0}^{v-1} \frac{1}{\left|e_{\alpha}\left(q^{j}\right)\right|}+\frac{\log _{q} t-v}{\mu_{3}}\right)
\end{aligned}
$$

for $t \geq q^{\nu}$. Hence we have $\lim _{t \rightarrow \infty} \gamma(t)=\infty$, so

$$
|\zeta(t)-z(t)|=\left|\gamma(t)-z_{0}\right|\left|e_{\alpha}(t)\right| \geq \mu_{2}\left|\gamma(t)-z_{0}\right| \rightarrow \infty
$$

for any $z_{0} \in \mathbb{C}$, and (1.1) is not Ulam stable.

Corollary 4.3 Let $\alpha(t)$ satisfy (1.2), (2.4), and (4.3). If $\beta=\frac{1}{2}$, then (1.1) is unstable in the Ulam sense.

Proof If $\beta=\frac{1}{2}$, then $e_{\alpha}(t)$ is given by

$$
e_{\alpha}(t)=\prod_{j=0}^{\log _{q} t-1} \frac{1+\frac{1}{2}(q-1) q^{j} \alpha\left(q^{j}\right)}{1-\frac{1}{2}(q-1) q^{j} \alpha\left(q^{j}\right)} .
$$

Conditions (1.2) and (2.4) imply that $e_{\alpha}(t)$ converges to a two-cycle $\pm \xi^{*}$ for some $\xi^{*} \in$ $\mathbb{C} \backslash\{0\}$ as $t \rightarrow \infty$. Hence (4.4) holds. Then, by Theorem 4.2, (1.1) is not Ulam stable.

Remark 4.4 Theorem 4.2 implies the instability result for the constant coefficient case given in [8, Theorem 3.1]. Indeed, consider the case $\alpha(t) \equiv \alpha$ with (1.2). If $\alpha \neq 0$, then Corollary 4.3 immediately shows instability. If $\alpha=0$, then $e_{\alpha}(t) \equiv 1$, and (4.4) holds. Hence, by Theorem 4.2, (1.1) is not Ulam stable.

\section{Conclusions}

Using the properties of the exponential function for nonautonomous Cayley quantum equations, we established sufficient conditions for the Ulam stability of quantum equations with a variable coefficient under the assumptions that the Cayley parameter satisfies $\beta \neq \frac{1}{2}$ and the absolute value of the variable coefficient does not approach zero. After that, these assumptions are elaborated. The situation is clarified by presenting an example where Ulam stability breaks down if the absolute value of the variable coefficient approaches zero. If the coefficient is a constant, it has already been shown in [8] that $\beta=\frac{1}{2}$ means the Ulam instability, but with the variable coefficient, something interesting happens, that is, if the absolute value of the variable coefficient increases, the Ulam stability 
is derived. Therefore it turns out that both Ulam stable and unstable cases may occur for $\beta=\frac{1}{2}$. In this way, we found in this study that by considering variable coefficients there is a problem of balance between stability and instability, which does not appear in the case of constant coefficients.

\section{Acknowledgements}

Both authors would like to thank the referees for their helpful suggestions.

Funding

MO was supported by JSPS KAKENHI Grant Number JP20K03668.

Availability of data and materials

Not applicable.

\section{Competing interests}

The authors declare that they have no competing interests.

\section{Authors' contributions}

Both authors contributed equally to this work. Both authors read and approved the final manuscript.

\section{Author details}

${ }^{1}$ Department of Mathematics, Concordia College, Moorhead, MN 56562, USA. ²Department of Applied Mathematics, Okayama University of Science, Okayama, 700-0005, Japan.

\section{Publisher's Note}

Springer Nature remains neutral with regard to jurisdictional claims in published maps and institutional affiliations.

Received: 23 June 2021 Accepted: 21 September 2021 Published online: 29 September 2021

\section{References}

1. Abbas, S., Benchohra, M., Laledj, N., Zhou, Y.: Existence and Ulam stability for implicit fractional $q$-difference equations. Adv. Differ. Equ. 2019, 480 (2019). https://doi.org/10.1186/s13660-019-2257-6

2. Abbas, S., Benchohra, M., Samet, B., Zhou, Y.: Coupled implicit Caputo fractional q-difference systems. Adv. Differ. Equ. 2019, 527 (2019). https://doi.org/10.1186/s13662-019-2433-5

3. Abdollahpour, M.R., Aghayari, R., Rassias, M.T.: Hyers-Ulam stability of associated Laguerre differential equations in a subclass of analytic functions. J. Math. Anal. Appl. 437(1), 605-612 (2016). https://doi.org/10.1016/j.jmaa.2016.01.024

4. Abdollahpour, M.R., Rassias, M.T.: Hyers-Ulam stability of hypergeometric differential equations. Aequ. Math. 93(4), 691-698 (2019). https://doi.org/10.1007/s00010-018-0602-3

5. Aczél, J., Dhombres, J.: Functional Equations in Several Variables. With applications to mathematics, information theory and to the natural and social sciences. Encyclopedia of Mathematics and Its Applications, vol. 31. Cambridge University Press, Cambridge (1989). https://doi.org/10.1017/CBO9781139086578

6. Anderson, D.R., Onitsuka, M.: Hyers-Ulam stability and best constant for Cayley $h$-difference equations. Bull. Malays. Math. Sci. Soc. 43, 4207-4222 (2020). https://doi.org/10.1007/s40840-020-00920-z

7. Anderson, D.R., Onitsuka, M.: Hyers-Ulam stability for quantum equations of Euler type. Discrete Dyn. Nat. Soc. 2020, 5626481 (2020). https://doi.org/10.1155/2020/5626481

8. Anderson, D.R., Onitsuka, M.: Hyers-Ulam stability for quantum equations. Aequ. Math. 95(2), 201-214 (2021). https://doi.org/10.1007/s00010-020-00734-1

9. Anderson, D.R., Onitsuka, M.: Hyers-Ulam stability for Cayley quantum equations and its application to $h$-difference equations. Mediterr. J. Math. 18, 168 (2021). https://doi.org/10.1007/s00009-021-01794-6

10. Anderson, D.R., Onitsuka, M.: Best constant for Hyers-Ulam stability of two step sizes linear difference equations. J. Math. Anal. Appl. 496(2), 124807 (2021). https://doi.org/10.1016/j.jmaa.2020.124807

11. Anderson, D.R., Onitsuka, M., Rassias, J.M.: Best constant for Ulam stability of first-order $h$-difference equations with periodic coefficient. J. Math. Anal. Appl. 491 (2), 124363 (2020). https://doi.org/10.1016/j.jmaa.2020.124363

12. Baias, A.-R., Blaga, F., Popa, D.: On the best Ulam constant of a first order linear difference equation in Banach spaces. Acta Math. Hung. 163, 563-575 (2021). https://doi.org/10.1007/s10474-020-01098-3

13. Baias, A.-R., Popa, D.: On Ulam stability of a third order linear difference equation in Banach spaces. Aequ. Math. 94(6), 1151-1170 (2020). https://doi.org/10.1007/s00010-020-00722-5

14. Baias, A.-R., Popa, D.: On the best Ulam constant of a higher order linear difference equation. Bull. Sci. Math. 166 102928 (2021). https://doi.org/10.1016/j.bulsci.2020.102928

15. Baias, A.-R., Popa, D., Raşa, I.: Ulam stability of a successive approximation equation. J. Fixed Point Theory Appl. 22(2), 41 (2020). https://doi.org/10.1007/s11784-020-00777-6

16. Brzdęk, J., Wójcik, P.: On approximate solutions of some difference equations. Bull. Aust. Math. Soc. 95(3), 476-481 (2017). https://doi.org/10.1017/S0004972716001076

17. Buşe, C., Lupulescu, V., O'Regan, D.: Hyers-Ulam stability for equations with differences and differential equations with time-dependent and periodic coefficients. Proc. R. Soc. Edinb., Sect. A 150(5), 2175-2188 (2020). https://doi.org/10.1017/prm.2019.12

18. Buşe, C., O'Regan, D., Saierli, O.: Hyers-Ulam stability for linear differences with time dependent and periodic coefficients. Symmetry 11, 512 (2019). https://doi.org/10.3390/sym11040512 
19. Butt, R.I., Abdeljawad, T., Alqudah, M.A., ur Rehman, M.: Ulam stability of Caputo q-fractional delay difference equation: q-fractional Gronwall inequality approach. J. Inequal. Appl. 2019, 305 (2019). https://doi.org/10.1186/s13660-019-2257-6

20. Czerwik, S.: On the stability of the quadratic mapping in normed spaces. Abh. Math. Semin. Univ. Hamb. 62, 59-64 (1992). https://doi.org/10.1007/BF02941618

21. Czerwik, S.: Functional Equations and Inequalities in Several Variables. World Scientific, River Edge (2002). https://doi.org/10.1142/4875

22. Gajda, Z:: On stability of additive mappings. Int. J. Math. Math. Sci. 14(3), 431-434 (1991). https://doi.org/10.1155/S016117129100056X

23. Hyers, D.H., Isac, G., Rassias, T.M.: Stability of Functional Equations in Several Variables. Progress in Nonlinear Differential Equations and Their Applications, vol. 34. Birkhäuser Boston, Boston (1998)

24. Hyers, D.H., Rassias, T.M.: Approximate homomorphisms. Aequ. Math. 44(2-3), 125-153 (1992) https://doi.org/10.1007/BF01830975

25. Jung, S.-M.: Hyers-Ulam-Rassias Stability of Functional Equations in Nonlinear Analysis. Springer Optimization and Its Applications, vol. 48. Springer, New York (2011). https://doi.org/10.1007/978-1-4419-9637-4

26. Jung, S.-M., Nam, Y.W.: Hyers-Ulam stability of Pielou logistic difference equation. J. Nonlinear Sci. Appl. 10, 3115-3122 (2017). https://doi.org/10.22436/jnsa.010.06.26

27. Jung, S.-M., Popa, D., Rassias, M.T.: On the stability of the linear functional equation in a single variable on complete metric groups. J. Glob. Optim. 59(1), 165-171 (2014). https://doi.org/10.1007/s10898-013-0083-9

28. Jung, S.-M., Rassias, M.T.: A linear functional equation of third order associated with the Fibonacci numbers. Abstr. Appl. Anal. 2014, Article ID 137468 (2014). https://doi.org/10.1155/2014/137468

29. Jung, S.-M., Rassias, M.T., Mortici, C.: On a functional equation of trigonometric type. Appl. Math. Comput. 252 294-303 (2015). https://doi.org/10.1016/j.amc.2014.12.019

30. Kannappan, P.: Functional Equations and Inequalities with Applications. Springer Monographs in Mathematics. Springer, New York (2009). https://doi.org/10.1007/978-0-387-89492-8

31. Lee, Y.-H., Jung, S.-M., Rassias, M.T.: On an $n$-dimensional mixed type additive and quadratic functional equation. Appl. Math. Comput. 228, 13-16 (2014) (English summary). https://doi.org/10.1016/j.amc.2013.11.091

32. Lee, Y.-H., Jung, S.-M., Rassias, M.T.: Uniqueness theorems on functional inequalities concerning cubic-quadratic-additive equation. J. Math. Inequal. 12(1), 43-61 (2018). https://doi.org/10.7153/jmi-2018-12-04

33. Mortici, C., Rassias, M.T., Jung, S.-M.: On the stability of a functional equation associated with the Fibonacci numbers. Abstr. Appl. Anal. 2014, Article ID 546046 (2014). https://doi.org/10.1155/2014/546046

34. Nam, Y.W.: Hyers-Ulam stability of hyperbolic Möbius difference equation. Filomat 32(13), 4555-4575 (2018). https://doi.org/10.2298/FIL1813555N

35. Nam, Y.W.: Hyers-Ulam stability of elliptic Möbius difference equation. Cogent Math. Stat. 5(1), 1-9 (2018) https://doi.org/10.1080/25742558.2018.1492338

36. Nam, Y.W.: Hyers-Ulam stability of loxodromic Möbius difference equation. Appl. Math. Comput. 356, 119-136 (2019). https://doi.org/10.1016/j.amc.2019.03.033

37. Novac, A., Otrocol, D., Popa, D.: Ulam stability of a linear difference equation in locally convex spaces. Results Math. 76(1), 33 (2021). https://doi.org/10.1007/s00025-021-01344-2

38. Onitsuka, M.: Influence of the step size on Hyers-Ulam stability of first-order homogeneous linear difference equations. Int. J. Difference Equ. 12(2), 281-302 (2017)

39. Popa, D.: Hyers-Ulam stability of the linear recurrence with constant coefficients. Adv. Differ. Equ. 2005, 407076 (2005). https://doi.org/10.1155/ADE.2005.101

40. Popa, D.: Hyers-Ulam-Rassias stability of a linear recurrence. J. Math. Anal. Appl. 309, 591-597 (2005). https://doi.org/10.1016/j.jmaa.2004.10.013

41. Rasouli, H., Abbaszadeh, S., Eshaghi, M.: Approximately linear recurrences. J. Appl. Anal. 24(1), 81-85 (2018), https://doi.org/10.1515/jaa-2018-0008

42. Rassias, T.M.: On the stability of the linear mapping in Banach spaces. Proc. Am. Math. Soc. 72(2), 297-300 (1978). https://doi.org/10.1090/S0002-9939-1978-0507327-1

43. Rassias, T.M.: Functional Equations and Inequalities. Mathematics and Its Applications, vol. 518. Kluwer Academic, Dordrecht (2000). https://doi.org/10.1007/978-94-011-4341-7

44. Sahoo, P.K., Kannappan, P.: Introduction to Functional Equations. CRC Press, Boca Raton (2011)

45. Shen, Y., Li, Y.: The $z$-transform method for the Ulam stability of linear difference equations with constant coefficients. Adv. Differ. Equ. 2018, 396 (2018). https://doi.org/10.1186/s13662-018-1843-0

46. Wang, J.: Some further generalizations of the Hyers-Ulam-Rassias stability of functional equations. J. Math. Anal. Appl. 263(2), 406-423 (2001). https://doi.org/10.1006/jmaa.2001.7587 